Received 1 August 2019 Accepted 6 December 2019

Link to DOI:

10.25220/WNJ.V03.i2.0003

Journal Website: www.worldnutrijournal.org

\section{The Impact of Nutritional Status and Body Mass Index on Rehabilitation Outcomes in Patients Receiving Home-based Medical Care}

\author{
Kanako Eiwa ${ }^{1}$, Naomi Nakayama ${ }^{2 *}$, Yumi Takami $^{1}$, Shuko Iwasaki ${ }^{1}$, Yoshinori Hino ${ }^{1}$, Takehiko \\ Hirai $^{1}$, Kentaro Nakayama ${ }^{3}$, Yuji Eda ${ }^{1}$
}
1. Division of Home Visiting Rehabilitation, Eda Clinic, Shimane Prefecture, Japan
2. Department of Nursing and Nutrition, University of Shimane, Shimane Prefecture, Japan
3. Department of Obstetric and Gynaecology, Shimane University School of Medicine, Shimane Prefecture, Japan

\begin{abstract}
Background: Home-based medical care is expanding rapidly in Japan.

Objectives: We aimed to identify the factors associated with outcomes of therapy in patients receiving home-visit rehabilitation.

Methods: One hundred twenty-one patients receiving home-based rehabilitation were investigated. Nutritional status was assessed by the Mini Nutritional Assessment Short Form (MNA-SF). The Functional Independence Measure (FIM) was employed to assess the activities of daily living (ADL). The body mass index (BMI), medical history, and orthopaedic disease-related pain were also recorded. The primary outcome was the improvement in FIM scores in one year.

Results: A total of $19(17 \%)$ patients were malnourished and 58 (48\%) were at risk of malnutrition. Malnourished patients had a lower FIM score at initiation than those at risk of malnutrition or with normal nutritional status. Only changes in patients' BMI and MNASF scores over one year were significantly associated with improved FIM scores $(p=0.0079$ and $p=0.0049$, respectively). No association was noted with the other factors.

Conclusions: This is the first report to demonstrate that changes in MNA-SF scores and $\mathrm{BMI}$ are significantly associated with rehabilitation outcomes in home-based care. Nutritional management is essential along with rehabilitation to improve ADL in the longterm home care setting.
\end{abstract}

Keywords home-based medical care, nutritional status, body mass index

\section{Corresponding author:}

* Naomi Nakayama, MD, PhD,

Department of Nursing and Nutrition,

University of Shimane

151 Nishihayashigi-cho, Izumo, Shimane

6938550, Japan.

PHONE: +81-853-20-0200; FAX: +81-853-20-

0201

Email: kennaonatsuno@yahoo.co.jp

\section{Introduction}

The number of patients receiving home-based medical care in Japan has been steadily on the rise. ${ }^{1}$ This is primarily owing to the rapid growth of the older adult population in Japan, which has led to remarkable increases in medical expenses, and subsequent reductions in hospital-based medical care. 
Rehabilitation is the medical care intended to improve quality of life (QoL) in patients with impaired ADL, either due to disease, or disuse. Improvement of ADL is an essential part of acute and convalescent hospital care. Since most patients are able to receive hospital care only for a limited period of time, rehabilitation needs to be continued in the home-based setting. Rehabilitation practiced in the home care setting is offered by visiting therapists. Patients suitable for home- based medical care are either late in the convalescent phase, or in a chronic phase, and are unable to commute to the hospital or neighbourhood clinic due to several reasons including impaired mobility, and severe illness. Consequently, home rehabilitation, intended to improve or maintain $\mathrm{ADL}$, is essential for enhancing social activity, and QoL. In contrast to hospital care, home based medical care is a relatively long- term commitment, which also includes management in the terminal stages.

Malnutrition is common in older people receiving care in long-term care facilities, with a prevalence between $12 \%$ and $54 \% .{ }^{2}$ The prevalence varies, with considerable differences depending on the settings. The prevalence of malnutrition in rehabilitation facilities, general hospitals, nursing homes, and in the community, being $50.5 \%, 38.7 \%$, $13.8 \%$, and $5.8 \%$, respectively. ${ }^{3}$ According to our previous report, the prevalence of malnutrition in the home-care setting is $34 \%$, rising to $75 \%$ when at-risk patients are included. ${ }^{4}$ Rehabilitation outcomes have been known to be poorer in the hospital setting, among malnourished patients with stroke, chronic heart failure, chronic obstructive pulmonary disease, and a variety of other diseases. ${ }^{5,6}$ In a cohort of patients who received rehabilitation in the convalescent ward due to hospital-associated deconditioning, poor rehabilitation outcomes were found in $91 \%$ of malnourished patients, which was worse than that of patients with normal nutrition. ${ }^{7}$

Although the number of patients receiving home- rehabilitation has been increasing in Japan's current healthcare scenario, no reports are available from this setting. Therefore, the present study aimed to investigate rehabilitation outcomes, and their relationship with nutritional, and other factors, in patients from the long term home-based medical care setting.

\section{Materials and Methods}

This was a retrospective, observational study, conducted on patients who received rehabilitation in home-based medical care settings. All therapists involved in this study were from the single Japanese orthopaedic medical corporation, which has a home visiting rehabilitation division, with 34 home-visit therapists engaged in home-rehabilitation therapy. In order to identify the factors associated with outcomes of long term home rehabilitation, 121 patients who received home rehabilitation, continuously, for one year in the past two years (between January 2, 2016 and January 2, 2018), were analyzed.

The patients were prescribed physical therapy once or twice a week, including motion exercises, resistance training, physical restoration, movement exercises, and ambulation exercises at home. Each session of physical therapy was equal to 1 to 2 units ( 1 rehabilitation unit equated to 20 minutes of therapy). The nutritional status was assessed by the Mini Nutritional Assessment ShortForm (MNA-SF) at initiation of home rehabilitation, and after one year. ${ }^{8,9}$ The MNA-SF comprises six questions that address: (1) the decline in food intake over the past 3 months, (2) weight loss over the past 3 months, (3) mobility, (4) psychological stress or acute disease in the past 3 months, (5) neuropsychological problems, and (6) the body mass index. The ADL were evaluated by the Functional Independence Measure (FIM). ${ }^{10}$ The FIM consists of 18 items composed of 13 motor tasks, namely, eating, grooming, bathing, upper body dressing, lower body dressing, toileting, bladder management, bowel management, bed-to-chair transfer, toilet transfer, shower transfer, locomotion (ambulatory or wheelchair level), and negotiating stairs. It also includes 5 cognitive tasks, namely, cognitive comprehension, expression, social interaction, problem-solving, and memory. The tasks are rated on a 7- point ordinal scale, which ranges from total assistance, scored 0 , to complete independence, scored 7 . The scores range from 18 to 126 , according to the independence of the functional level. Patients with FIM scores higher than 108 are considered to be independent, whether they extra time, or a support device. ${ }^{11}$ The BMI, main basal disease, history of musculoskeletal disease, existence of 
orthopaedic pain, and feeding route (oral intake, enteral nutrition, or parenteral nutrition), were obtained from the interview and the medical records.

The outcome of home rehabilitation was classified into three categories, improved, maintained, and deteriorated, in accordance with the differences of the FIM score between point of initiation, and one year later. The patients were then divided into two groups, the "improved or maintained" group, and the "deteriorated" group. The patients-related factors were then analyzed to identify the factor that was related to the FIM score. Statistical analysis was performed using SPSS for Windows, Version 24 (IBM SPSS, Inc., Chicago, IL, USA). The patients' characteristics were compared using a chi-square test, as appropriate. A $\mathrm{p}$ value $<0.05$ was considered statistically significant.

\section{Results}

During the study period, 121 patients including 58 men, and 63 women, continuously received home rehabilitation, for longer than one year. The patients' characteristics are summarized in Table 1. Eleven patients $(11 \%)$ were younger than 65 years. The remaining 110 patients $(91 \%)$ were older than 65 years, with an average age of 79 . The basal diseases in this cohort were mostly various chronic conditions. The sequelae of strokes were most common in this group, followed by Parkinson's disease, and Alzheimer's disease. Around half of the patients had a history of musculoskeletal conditions, including vertebral compression fractures, hip fractures, and knee arthroplasty. More than half of the patients experienced orthopaedic pain at the point of initiation. The level of pain was evaluated by the visual analogue scale (VAS), and the pain in all patients was not continuous, did not exceed a score of 5 on the VAS, and was self-controlled.

The patients were classified into three groups according to their nutritional status, as assessed by the MNA-SF score (Table 1, 2). Patients with scores of 0-7 were considered to have malnutrition; those with scores of 8-11 were considered at risk of malnutrition, and those with scores of $12-15$, were considered to have normal nutrition. At the point of initiation, the prevalence of malnutrition in this cohort was $17 \%$, which rose to $22 \%$ in one year.
Malnourished patients showed lower BMI and FIM scores, whereas that of patients with better nutritional status was higher (Table 2). The outcomes of continuous 1-year home rehabilitation, is shown in Figure 1. An improvement in the FIM score was noted in 40 patients $(34 \%)$, while 21 $(16 \%)$ maintained their scores. Deterioration of existing scores was noted in $60(50 \%)$ patients.

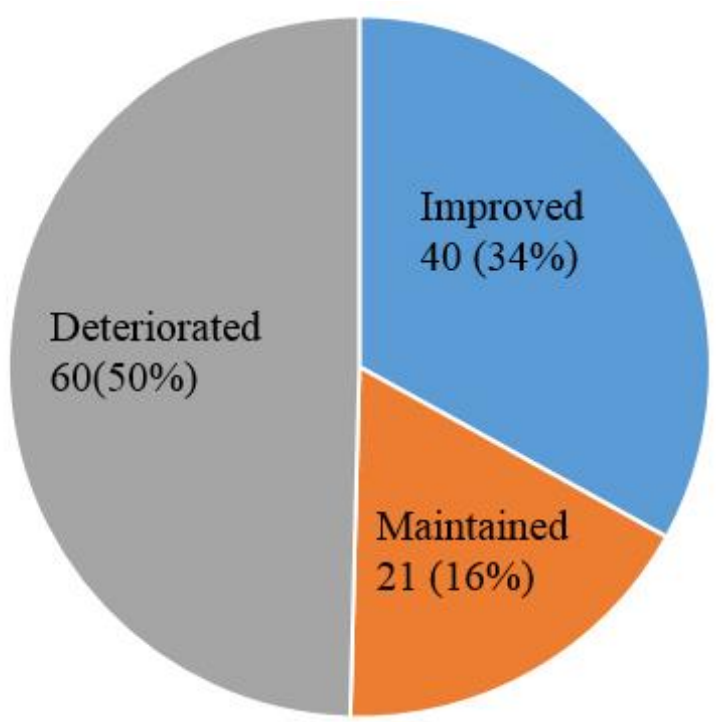

Figure 1. Rehabilitation outcomes after continuous 1 -year home-based care $(n=121)$

We subsequently investigated the patient-factors associated with the rehabilitation- outcomes. As shown in table 3, a change in BMI, and MNA-SF scores, were the factors that were associated with rehabilitation-outcomes. The existence of orthopaedic pain, the age, the gender, the BMI, the FIM scores, and the MNA-SF scores at point of initiation were not found to be associated with outcomes.

\section{Discussion}

Japan is undergoing a significant demographic shift, with a large section of adults in the older age group. In addition, birth rate is on the decline. This has led to increases in medical expenses, with considerable impact on the national social economy. Government policies have been implemented to reduce medical expenses, and to deal with this problematic demographic change. These policies include reduction in numbers of hospital beds, and shortening the lengths of hospital stay. 
Table 1a. Characteristics of subjects $(\mathrm{n}=121)$

\begin{tabular}{|c|c|}
\hline Characteristics & Numbers \\
\hline Age (y), average (SD) & $79 \pm 10$ \\
\hline \multicolumn{2}{|l|}{ Gender, $\mathrm{n}(\%)$} \\
\hline Male & $58(48)$ \\
\hline Female & $63(52)$ \\
\hline \multicolumn{2}{|l|}{ Main basal disease, $\mathrm{n}(\%)$} \\
\hline Cardiovascular diseases & $13(11)$ \\
\hline Gastroenterological diseases & $3(3)$ \\
\hline Kidney and urological diseases & $8(7)$ \\
\hline Cerebral and neurological diseases & $61(50)$ \\
\hline Haematological diseases & $2(2)$ \\
\hline Respiratory diseases & $11(10)$ \\
\hline Metabolic diseases & $8(7)$ \\
\hline Gynaecological diseases & $2(2)$ \\
\hline Autoimmune disease & $4(3)$ \\
\hline No specific basal disease & $9(8)$ \\
\hline History of musculoskeletal disease $(+), \mathrm{n}(\%)$ & $61(50.4)$ \\
\hline Hip fractures & 12 \\
\hline Knee arthroplasty & 11 \\
\hline Vertebral compression fractures & 17 \\
\hline Spinal canal stenosis & 13 \\
\hline Other & 8 \\
\hline Orthopaedic pain $(+), \mathrm{n}(\%)$ & $82(67.7)$ \\
\hline MNA-SF at point of initiation, average, (SD) & $9.9 \pm 2.7$ \\
\hline Malnutrition, $\mathrm{n}(\%)$ & $19(17)$ \\
\hline At risk of malnutrition, $\mathrm{n}(\%)$ & $58(48)$ \\
\hline Normal nutrition status, n(\%) & $44(36)$ \\
\hline MNA-SF in one year, average, (SD) & $9.7 \pm 2.9$ \\
\hline Malnutrition, $\mathrm{n}(\%)$ & $27(22)$ \\
\hline At risk of malnutrition, $\mathrm{n}(\%)$ & $54(45)$ \\
\hline Normal nutrition status, $\mathrm{n}(\%)$ & $40(33)$ \\
\hline \multicolumn{2}{|l|}{ Feeding route, $\mathrm{n}(\%)$} \\
\hline Oral intake only & $3(2.4)$ \\
\hline Oral intake and enteral nutrition & $118(97.6)$ \\
\hline BMI at point of initiation, average, (SD) & $21 \pm 3.9$ \\
\hline BMI in one year, average, (SD) & $21 \pm 3.8$ \\
\hline FIM at point of initiation, average, (SD) & $100 \pm 25.8$ \\
\hline FIM in one year, average, (SD) & $97 \pm 27.4$ \\
\hline
\end{tabular}

Consequently, home-based medical care has been expanding rapidly, and the number of patients receiving home-based medical care is on the rise. Home care patients receive rehabilitation within their permitted insurance coverage; its duration is usually longer, and frequency lower, than the intensive rehabilitation provided by hospital care. In general, home care patients regularly receive rehabilitation once or twice a week. The impaired ADL of elderly patients in long term care facilities have an adverse impact on their QoL. ${ }^{12}$ Home rehabilitation should therefore be considered as a key factor in improving or maintaining patients' ADL, while enhancing their social activities, which contribute to improved QoL.

Rehabilitation outcome in long term home settings is worse than hospital settings. Only $34 \%$ of patients rehabilitated at home showed an improvement in FIM scores, compared to $90 \%$ in the convalescent hospital setting. ${ }^{13}$ As we focused on evaluating outcomes of only long-term homerehabilitation in this study, patients who received home rehabilitation for less than one year were excluded. A total of 210 patients attending our clinic 
Table 1b. Details of main basal diseases $(n=121)$

\begin{tabular}{|c|c|}
\hline \multicolumn{2}{|l|}{ Main basal diseases, $\mathrm{n}(\%)$} \\
\hline Cardiovascular disease & $13(11)$ \\
\hline Chronic heart failure & 6 \\
\hline Chronic artrial flutter & 2 \\
\hline Hypertension & 3 \\
\hline Other cardiovascular diseases & 2 \\
\hline Gastroenterological diseases & $3(3)$ \\
\hline Hepatocellular carcinoma & 1 \\
\hline Cholangiocarcinoma & 1 \\
\hline Gastric cancer & 1 \\
\hline Kidney and urological diseases & $8(7)$ \\
\hline Chronic renal failure & 5 \\
\hline Other kidney and urological diseases & 3 \\
\hline Cerebral and neurological diseases & $61(50)$ \\
\hline Alzheimer's disease & 8 \\
\hline Parkinson's disease & 12 \\
\hline Sequelae of strokes & 33 \\
\hline Guillain-Barre syndrome & 4 \\
\hline Other neurological disease & 4 \\
\hline Haematological diseases & $2(2)$ \\
\hline MDS (myelodysplastic syndromes) & 1 \\
\hline Malignant lymphoma & 1 \\
\hline Respiratory diseases & $11(10)$ \\
\hline Interstitial pneumonia & 1 \\
\hline Lung cancer & 3 \\
\hline COPD (chronic obstructive pulmonary disease) & 1 \\
\hline Other respiratory disease & 4 \\
\hline Metabolic disease & $8(7)$ \\
\hline Diabetes mellitus & 7 \\
\hline Hyperlipidemia & 1 \\
\hline Gynecological disease & $2(2)$ \\
\hline Uterus cancer & 2 \\
\hline Autoimmune disease & $4(3)$ \\
\hline SLE (systemic lupus erythematosus) & 2 \\
\hline RA (rheumatoid arthritis) & 2 \\
\hline No specific basal disease & $9(8)$ \\
\hline
\end{tabular}

Table 2. Nutritional status and average of FIM and BMI

\begin{tabular}{llll}
\hline MNA-SF at point of initiation & $\mathrm{n}(\%)$ & FIM, average(SD) & BMI, average(SD) \\
\hline Malnutrition & $19(17)$ & $83 \pm 33$ & $18 \pm 2$ \\
At risk of malnutrition & $58(48)$ & $98 \pm 26$ & $20 \pm 3$ \\
Normal nutrition status & $44(36)$ & $110 \pm 15$ & $24 \pm 2$ \\
& & & \\
\hline MNA-SF in one year & $\mathrm{n}(\%)$ & FIM, average(SD) & BMI, average(SD) \\
\hline Malnutrition & $27(22)$ & $75 \pm 33$ & $18 \pm 2$ \\
At risk of malnutrition & $54(45)$ & $102 \pm 22$ & $20 \pm 3$ \\
Normal nutrition status & $40(33)$ & $107 \pm 20$ & $25 \pm 3$ \\
\hline
\end{tabular}

received home rehabilitation during the two-year observation period. Among them, 121 received continuous rehabilitation for longer than one year, while 89 received shorter rehabilitation care, lasting less than one year. A total of 44/89 short-term care patients demonstrated improved FIM scores, and had discontinued home-based care, as either their rehabilitation goals were accomplished, or they had 
Table 3. Analysis of the factors associated with home rehabilitation outcome

\begin{tabular}{|c|c|c|c|c|}
\hline & & \multicolumn{2}{|c|}{ FIM } & \multirow[b]{2}{*}{$\mathrm{p}$ value } \\
\hline & & Improved group $(\mathrm{n}=41)$ & No-improvement group $(\mathrm{n}=80)$ & \\
\hline \multirow[t]{2}{*}{ Age } & $80>$ & 24 & 33 & \multirow{2}{*}{0.714} \\
\hline & $80<$ & 17 & 47 & \\
\hline \multirow[t]{2}{*}{ Gender } & Male & 22 & 36 & \multirow{2}{*}{0.3669} \\
\hline & Female & 19 & 44 & \\
\hline \multirow[t]{2}{*}{ Orthopedic pain } & No & 12 & 27 & \multirow{2}{*}{0.6176} \\
\hline & Yes & 29 & 53 & \\
\hline \multirow{2}{*}{ FIM at the point of initiation } & $108<$ & 21 & 46 & \multirow{2}{*}{0.6887} \\
\hline & $108>$ & 20 & 34 & \\
\hline \multirow[t]{2}{*}{$\mathrm{BMI}$ at the point of initiation } & $20<$ & 29 & 58 & \multirow{2}{*}{0.5107} \\
\hline & $20>$ & 13 & 22 & \\
\hline \multirow[t]{2}{*}{ BMI change } & no change & 28 & 33 & \multirow[t]{2}{*}{0.0079} \\
\hline & Decrease & 10 & 37 & \\
\hline \multirow[t]{2}{*}{ MNA at the point of initiation } & $8 \sim 14$ & 26 & 43 & \multirow{2}{*}{0.3094} \\
\hline & $0 \sim 7$ & 15 & 37 & \\
\hline \multirow[t]{2}{*}{ MNA in one year } & $8 \sim 14$ & 32 & 53 & \multirow{2}{*}{0.179} \\
\hline & $0 \sim 7$ & 9 & 27 & \\
\hline \multirow[t]{2}{*}{ MNA change } & no change & 29 & 35 & \multirow{2}{*}{0.0049} \\
\hline & terioration & 12 & 45 & \\
\hline
\end{tabular}

been transferred to the outpatient rehabilitation services. Considering the entire patient cohort, regardless of the rehabilitation period, outcomes were better, with $40 \%$ showing improved FIM scores. The efficacy of physical rehabilitation is not the same in the different phases of rehabilitation therapy. In Japan, owing to the insurance system, cases of stroke or orthopaedic diseases receive acute rehabilitation for two weeks in the acute hospital setting, followed by convalescent rehabilitation for a maximum of six months in the convalescent hospital setting. ${ }^{14}$ Most of the recovery of patients' ADL usually occurs in the acute and convalescent phase, with little recovery during the maintenance phase, which is a part of home rehabilitation. Compared to long term care facilities, stroke patients in acute and convalescent hospitals have a higher rate of recovery, as evidenced by improved FIM scores. ${ }^{15}$

Malnutrition is associated with poorer rehabilitation outcomes and physical function in patients with stroke, hip fracture, hospital-associated deconditioning, and a variety of other diseases. ${ }^{16}$ The MNA-SF score, and the serum albumin level, have also shown to be significantly associated with ADLs in older in-patients, with hospital-associated deconditioning. ${ }^{17}$ In general, the nutritional status at first screening is strongly associated with rehabilitation outcomes in the hospital setting. ${ }^{17}$ However, in the present study, the nutritional status, BMI, and FIM at point of initiation, were not associated with rehabilitation outcomes in the longterm home care setting. This was probably because the nutritional status and the ADL scores at point of initiation are better in home-care patients, compared those in the hospital setting. The prevalence of malnutrition is much higher in hospital patients than in home care patients, the rates being $38.7 \%$ and $17 \%$, respectively. ${ }^{3}$ The average total FIM scores in the convalescent hospital and long term home care settings are 72 , and 100 , respectively. ${ }^{18}$ A study showed that gains of more than 20 points in FIM 
scores were noted in $51.4 \%$ of patients in the convalescent hospital setting, compared to only $2.4 \%$ among those receiving long term home care. ${ }^{13}$ Consequently, the association between ADL improvements and patient-related factors are not evident in long term home care. Our results showed that changes in the MNA-SF score, and the BMI were significantly associated with rehabilitation outcomes. Deterioration of the nutritional status and loss of body weight were associated with poorer outcomes. It also indicated that, an improvement in patients' ADL may be expected, regardless of the independence level, and the nutritional status at point of initiation. Monitoring the nutritional status, and maintenance of the body weight are both important in improving the ADL by home rehabilitation, in the long term home care setting. These factors are also important in the hospital setting to ensure better rehabilitation outcomes. Nutritional supplements have been associated with improved outcomes in post-stroke rehabilitation. ${ }^{19}$ Patients receiving intensive nutritional supplementation have shown more improvement on scores of total FIM. ${ }^{19}$ A randomized controlled trial in acute stroke patients at nutritional risk, compared routine care with individualized nutritional care to prevent weight loss. The results showed that $20.7 \%$ of the intervention group lost at least $5 \%$ body weight compared with $36.4 \%$ of the control group. ${ }^{20}$ Nutritional intervention with resistance training during convalescent rehabilitation has been shown to improve skeletal muscle mass, volume, and ADL, in older patients. ${ }^{21}$ About $70 \%$ of older people living in long term care facilities are malnourished; malnutrition, and total FIM score, were independently associated with the time taken to return home. ${ }^{22}$ Reports from literature confirm the findings of this study, demonstrating the benefits of nutritional support on rehabilitation outcomes in patients in the hospital and home care setting.

Interdisciplinary nutrition management is recommended for patients who receive rehabilitation therapy in hospital setting. ${ }^{16}$ The term "rehabilitation nutrition" has been coined based on the combination of both, rehabilitation, and nutrition care management. ${ }^{16}$ It emphasizes the need for proper evaluation of the nutritional status, and the implementation of nutritional management, to maximize the efficacy of rehabilitation. It enhances the recovery of functionality in the elderly, and the disabled. ${ }^{16}$ Management by a dedicated and specialized team has a positive impact on rehabilitation- nutrition, and this team-based medical practice has been expanding within the hospital setting. ${ }^{23}$ However, the practice of management by a nutrition support team (NST) has just started in the home care setting, and only few reports are available on the efficacy of nutritional improvement in home-care patients [4]. In order to enhance the efficacy of rehabilitation in long term home care, nutrition care management, which is provided by a specialized team, is essential.

This study has some limitations. Firstly, laboratory data, including levels of albumin, haemoglobin, and cholesterol, were not analyzed in the present study, because therapists do not perform blood tests. There is a possibility of association between these biomarkers and home- rehabilitation outcomes. Secondly, we analyzed the rehabilitation outcome using the total FIM score, as the total FIM comprehensively reflects the patients' level of independence. We did not analyze motor-FIM, and cognitive-FIM separately in this study. Thirdly, the participants were limited to a single medical corporation. Cooperative, multi-institutional research will be required in the future to validate our findings.

\section{Conclusion}

In conclusion, home-rehabilitation outcomes in long-term home-based care settings are not as good as that of hospital-rehabilitation. A decrease in the body weight, and the MNA-SF score is associated with poor rehabilitation outcomes, in this setting. Nutritional management should be provided in conjunction with rehabilitation for better outcomes in the home care setting. Further research is needed on the effect of home NST, and multidisciplinary nutritional management, on rehabilitation outcomes.

\section{Conflict of Interest}

Statement of Ethics

Written informed consent was obtained from all the patients for the publication of this paper.

The protocol and study design were approved to be ethically acceptable by the chairman of our medical institute. 
Disclosure statement

The authors have no conflicts of interest to declare.

\section{Funding Sources}

There is no funding source for this study

Author Contributions

$\mathrm{KE}$, and $\mathrm{NN}$ contributed equally. $\mathrm{NN}$ was responsible for the article planning, and the manuscript preparation. KE was responsible for the data collection. All authors have read and approved the final manuscript.

\section{Open Access}

This article is distributed under the terms of the Creative Commons Attribution 4.0 International Licence

(http://creativecommons.org/licenses/by/4.0/), which permits unrestricted use, distribution, and reproduction in any medium, provided you give appropriate credit to the original author(s) and the source, provide a link to the Creative Commons license, and indicate if changes were made.

\section{Reference}

1. Akiyama A, Hanabusa H, Mikami H. Trends associated with Home Care Supporting Clinics (HCSCs) in Japan. Arch GerontolGeriatr. 2012; 54(3): e383-6

2. Namasivayam AM, Steele CM. Malnutrition and Dysphagia in long-term care: a systematic review. J NutrGerontolGeriatr. 2015;34(1):1-21.

3. Kaiser MJ, Bauer JM, Rämsch C, Uter W, Guigoz Y, Cederholm T, Thomas DR, Anthony PS, Charlton KE, Maggio M, Tsai AC, Vellas B, Sieber CC; Mini Nutritional Assessment International Group. Frequency of malnutrition in older adults: a multinational perspective using the mini nutritional assessment. J Am Geriatr Soc. 2010 Sep;58(9):1734-8.

4. Nakayama N, Higashiguchi T, Maniwa A, Kanemoto Y, Hanada K, Sugiura H, Takami Y, Kumagai T, Hayash E, Fukuba E. The NST (Nutrition Support Team) practice and its efficacy in home-based medical care setting in Izumo. Journal of Japan society of parenteral and enteral nutrition, 2019 in press
5. Davis JP, Wong AA, Schluter PJ, Henderson RD, O'Sullivan JD, Read SJ. Impact of premorbid undernutrition on outcome in stroke patients. Stroke 2004; 35: 1930-1934.

6. Anker SD, John M, Pedersen PU, Raguso C, Cicoira M, Dardai E, et al. ESPEN guidelines on enteral nutrition: cardiology and pulmonology. ClinNutr 2006; 20: 311-318.

7. Wakabayashi H, Sashika H. Association of nutrition status and rehabilitation outcome in the disuse syndrome: a retrospective cohort study. Gen Med 2011; 12: 69-74.

8. Vellas B, Villars H, Abellan G, et al. Overview of the MNA-Its historyand challenges. J Nutr Health Aging. 2006;10:456-63; discussion 46365.

9. Guigoz Y. The Mini Nutritional Assessment (MNA) review of the literature-What does it tell us? J Nutr Health Aging. 2006;10:466-85

10. Ottenbacher KJ, Hsu Y, Granger CV, Fiedler $\mathrm{RC}$. The reliability of the functional independence measure: a quantitative review. Arch Phys Med Rehabil. 1996;77:1226 32.

11. Uemura S, Miki M. The characteristics of independent lives old solitude older females revealed by the comprehensive assessment using various scales. Human nursing research 2012, 10:25-31

12. Vitorino LM, Paskulin LM, Viana LA. Quality of life among older adults resident in long-stay care facilities. Rev Lat Am Enfermagem. 2012; 20: $1186-95$

13. The report from the general incorporated association of the convalescent rehabilitation http://plus1co.net/d_data/29_zitai_book.pdf201 7:108

14. Miyai I, Sonoda S, Nagai S, Takayama Y, Inoue Y, Kakehi A, et al. Results of new policies for inpatient rehabilitation coverage in Japan. Neurorehabil Neural Repair. 2011;25:540-7.

15. Black-Schaffer RM, Winston C. Age and functional outcome after stroke. Top Stroke Rehabil 2004; 11: 23-32

16. Wakabayashi H, Sakuma K. Rehabilitation nutrition for sarcopenia with disability: a combination of both rehabilitation and nutrition care management. J Cachexia Sarcopenia Muscle. 2014 Dec;5(4):269-77

17. Wakabayashi H, Sashika H. Malnutrition is associated with poor rehabilitation outcome in elderly inpatients with hospital-associated deconditioning a prospective cohort study. $\mathrm{J}$ Rehabil Med. 2014 Mar;46(3):277-82 
18. Shiraishi A, Yoshimura Y, Wakabayashi H, Tsuji Y. Prevalence of stroke-related sarcopenia and its association with poor oral status in postacute stroke patients: Implications for oral sarcopenia. ClinNutr. 2018 Feb;37(1):204-207

19. Rabadi MH, Coar PL, Lukin M, Lesser M, Blass JP. Intensive nutritional supplements can improve outcomes in stroke rehabilitation. Neurology. 2008;71:1856-61.

20. Ha L, Hauge T, Spenning AB, Iversen PO. Individual, nutritional support prevents undernutrition, increases muscle strength and improves QoL among elderly at nutritional risk hospitalized for acute stroke: a randomized, controlled trial. ClinNutr. 2010;29:567-73

21. Yoshimura Y, Uchida K, Jeong S, Yamaga M. Effects of Nutritional Supplements on Muscle Mass and Activities of Daily Living in Elderly
Rehabilitation Patients with Decreased Muscle Mass: A Randomized Controlled Trial. J Nutr Health Aging. 2016 Feb;20(2):185-91

22. Nishida Y, Wakabayashi H, Maeda K, Nishioka $\mathrm{S}$. Nutritional status is associated with the return home in a long-term care health facility. J Gen Fam Med. 2017 Nov 9;19(1):9-14

23. Kokura Y, Wakabayashi H, Maeda K, Nishioka S, Nakahara S. Impact of a multidisciplinary rehabilitation nutrition team on evaluating sarcopenia, cachexia and practice of rehabilitation nutrition. $\mathrm{J}$ Med Invest. 2017;64(1.2):140-145. 